Revue pluridisciplinaire d'études médiévales

\title{
Délimiter, exploiter et chasser : la forêt de Talmont et ses usages $\left(\mathrm{XII}^{\mathrm{e}}-\mathrm{XV}^{\mathrm{e}}\right.$ siècle)
}

\section{Charles Viaut}

\section{OpenEdition}

\section{Journals}

Édition électronique

URL : https://journals.openedition.org/questes/5789

DOI : 10.4000/questes.5789

ISSN : 2109-9472

\section{Éditeur}

Les Amis de Questes

\section{Édition imprimée}

Date de publication : 30 juillet 2021

Pagination : $37-55$

ISSN : 2102-7188

\section{Référence électronique}

Charles Viaut, "Délimiter, exploiter et chasser : la forêt de Talmont et ses usages ( $\|^{\mathrm{e}}{ }^{-x v^{e}}$ siècle) ",

Questes [En ligne], 43| 2021, mis en ligne le 30 octobre 2021, consulté le 08 décembre 2022. URL

http://journals.openedition.org/questes/5789; DOI : https://doi.org/10.4000/questes.5789 


\title{
Délimiter, exploiter et chasser : la forêt de Talmont et ses usages $\left(\mathrm{XII}^{\mathrm{e}}-\mathrm{XV}^{\mathrm{e}}\right.$ siècle)
}

\author{
Charles VIAUT \\ CESCM - Université de Poitiers
}

L'historiographie récente en histoire et archéologie de l'environnement tend à mettre en valeur les derniers siècles du Moyen Âge, ce dernier entendu dans la chronologie traditionnellement retenue $\left(\mathrm{V}^{\mathrm{e}}-\mathrm{XV}^{\mathrm{e}}\right.$ siècle $)$ comme une époque charnière dans l'anthropisation des milieux naturels. Les « forçages », pour reprendre la formule de Joëlle Burnouf ${ }^{1}$, approfondissent alors ceux des époques précédentes et entraînent des modifications irréversibles de certains environnements. On assiste, à la même époque, à la fixation des droits sur les ressources naturelles et à leur mise en forme écrite ${ }^{2}$. Les forêts, espaces supposément à l'écart, hors de la civilisation humaine, sont un lieu commun des fantasmes et de l'imaginaire, tant médiéval qu'actuel ${ }^{3}$. En témoigne sa place privilégiée tant dans les littératures médiévales que dans les fictions contemporaines prenant pour cadre le Moyen Âge européen. Dans l'historiographie française, l'histoire des forêts n'a trouvé sa place qu'au sein d'une histoire économique et sociale de plus en plus développée à partir des années 1950 et 1960. De fait, comme l'ont

\footnotetext{
${ }^{1}$ Joëlle Burnouf et al., "Sociétés, milieux, ressources : un nouveau paradigme pour les médiévistes » dans Être historien du Moyen Âge au XXI $I^{e}$ siècle. XXVIII ${ }^{\mathrm{e}}$ Congrès de la SHMESP (Cergy-Pontoise, Évry, Marne-la-Vallée, Saint-Quentin-en-Yvelines, 31 mai-3 juin 2007), Paris, Publications de la Sorbonne, 2007, p. 95-132, cit. p. 102.

${ }^{2}$ Fabrice Mouthon, Le sourire de Prométhée. L'homme et la nature au Moyen Âge, Paris, La Découverte, 2017, cit. p. 160.

${ }^{3}$ Voir notamment Robert Harrison, Forêts. Essai sur l'imaginaire occidental, Paris, Flammarion, 1992.
} 
notamment mis en lumière les recherches pionnières de Roland Bechmann en la matière ${ }^{4}$, les espaces boisés étaient essentiels à bien des égards à l'économie agricole du Moyen Âge européen; ils eurent ainsi tendance à concentrer les enjeux politiques et économiques à mesure que leur superficie régressait devant les assauts des hommes ${ }^{5}$.

L'exploration interdisciplinaire d'un cas particulier sur le temps long le montre bien : celui de la forêt de Talmont entre le XII ${ }^{\mathrm{e}}$ et le $\mathrm{XV}^{\mathrm{e}}$ siècle. Le passé médiéval de cet espace n'a attiré l'attention que récemment, dans la périphérie de recherches en cours sur d'autres thèmes. Le château de Talmont est un monument historique classé, dominant le petit bourg de Talmont Saint-Hilaire, en Vendée, au sud des Sables d'Olonne. Fondé probablement par le comte de Poitiers entre la fin du $\mathrm{X}^{\mathrm{e}}$ et le début $\mathrm{du} \mathrm{XI}^{\mathrm{e}}$ siècle, le château de Talmont est partiellement détruit puis abandonné au début $\mathrm{du} \mathrm{XVII}^{\mathrm{e}}$ siècle. Le site est investi depuis plusieurs années par un programme de recherches archéologiques et historiques soutenu par l'Université de Poitiers (laboratoire CESCM), l'Institut national de recherches archéologiques préventives (Inrap GrandOuest) et le département de la Vendée, et les résultats des investigations sont désormais en cours de publication ${ }^{6}$. La forêt s'est en quelque sorte « invitée » dans les recherches du fait de sa présence insistante dans la documentation. Elle est désormais considérée comme un élément d'une importance primordiale pour la compréhension des enjeux territoriaux,

\footnotetext{
${ }^{4}$ Roland Bechmann, Des arbres et des hommes : la forêt au Moyen Âge, Paris, Flammarion, 1984.

${ }^{5}$ Sur l'apport récent de l'archéologie et des sciences paléo-environnementales à la remise en cause des grands défrichements, voir notamment Fabrice Mouthon, Le sourire de Prométhée, op. cit., et Vincent Carpentier et Philippe Leveau, Archéologie du territoire de la France, 8000 ans d'aménagements, Paris, La Découverte, 2013.

${ }^{6}$ Teddy Béthus et Nicolas Prouteau, «Les mutations du castrum de Talmont (Vendée) : naissance et transformations d'une forteresse bas-poitevine ( $\mathrm{X}^{\mathrm{e}}-\mathrm{XIII}{ }^{\mathrm{e}}$ siècles) », dans Demeurer, défendre et paraître. Orientations récentes de l'archéologie des fortifications et des résidences aristocratiques médiévales entre Loire et Pyrénées, Chauvigny, Association de Publications Chauvinoises, 2014, p. 231-257 ; Charles Viaut, Du lieu de pouvoir à l'espace vécu : le château de Talmont du XIII au $X V^{e}$ siècle, thèse pour le diplôme d'archiviste-paléographe soutenue en 2018, Paris, École nationale des Chartes, 3 vol.
} 
économiques et politiques de la seigneurie de Talmont au Moyen Âge. Malgré tout, les recherches sur les environnements anciens et leur exploitation par les hommes n'en sont qu'à leurs balbutiements en Talmondais et nos réflexions reposent essentiellement sur les données textuelles. Celles-ci sont pourtant largement lacunaires, notamment pour les périodes antérieures au XII ${ }^{\mathrm{e}}$ siècle. Pour l'heure, il est seulement possible de dresser un premier bilan de la délimitation des milieux forestiers au Moyen Âge et des différentes formes prises par leur domestication en lien avec l'occupation castrale, monastique et paysanne du Talmondais.

\section{Retrouver les forêts médiévales}

Le Talmondais actuel (Vendée, arrondissement des Sables d'Olonne) est marqué, comme le reste des côtes vendéennes, par la périurbanisation et l'étalement des constructions pavillonnaires. Toutefois, le paysage agricole «traditionnel», selon la distinction de la géographie classique française $\mathrm{du} \mathrm{XX}^{\mathrm{e}}$ siècle, $\mathrm{y}$ est le bocage, paysage mixte de champs et de haies végétales associés à un habitat dispersé. La mise en place de ce paysage, jadis associé aux "grands défrichements » médiévaux voire aux époques plus anciennes, antiques et protohistoriques, est désormais retardée par les recherches les plus récentes à la fin du Moyen Âge et à l'époque moderne ${ }^{7}$. Le couvert forestier continu est quasiment absent du Talmondais actuel si l'on excepte quelques bois côtiers. Aujourd'hui, parler de « forêt de Talmont» ne correspond donc plus à aucune réalité paysagère ni même toponymique. Travailler sur la forêt de Talmont au Moyen Âge implique

\footnotetext{
7 Annie Antoine, Le paysage de l'historien. Archéologie des bocages de l'Ouest de la France à l'époque moderne, Rennes, Presses Universitaires de Rennes, 2002 ; Magali Watteaux, " Le bocage, un paysage rural à la lumière des études archéologiques et archéogéographiques ", Archéopages, $\mathrm{n}^{\circ} 34$, 2012, p. 64-73, cit. p. 72.
} 
dès lors d'étudier une forêt disparue et uniquement présente dans la documentation archéologique et historique. Le Talmondais n'a pour le moment fait l'objet d'aucune analyse palynologique ${ }^{8}$ portant sur le couvert végétal ancien mais les forêts ont laissé leur trace dans la documentation écrite et graphique, qui permet d'attester de leur existence à des périodes anciennes.

L'analyse régressive des documents cartographiques à notre disposition montrent pourtant qu'un certain couvert forestier a perduré dans la région jusqu'à l'aube de l'époque contemporaine. Le premier cadastre de 1828 montre un bocage achevé, sans plus aucune surface boisée d'importance à l'échelle du canton ${ }^{9}$. Sur la portion de la carte de Cassini concernant le littoral poitevin, dressée au milieu du XVIII ${ }^{\mathrm{e}}$ siècle, on décèle encore des espaces incultes d'importance, notamment à l'ouest du bourg actuel de Talmont ${ }^{10}$. La Carte generalle des costes du BasPoictou, pays d'Aunis, Saintonge et Isles adjacentes, Medoc et partie de celle de la Basse Guienne dressée par Claude Masse en $1719^{11}$ montre, elle aussi, un couvert forestier résiduel au nord et à l'ouest de Talmont. La documentation cartographique nous donne donc un terminus ante quem de la disparition de ce massif forestier. Que trouve-t-on à l'inverse dans la documentation la plus ancienne?

La forêt de Talmont fait son apparition dans la documentation avec les plus anciens textes médiévaux connus dans la région, $\mathrm{au} \mathrm{XI}^{\mathrm{e}}$ et au début $\mathrm{du} \mathrm{XII}^{\mathrm{e}}$ siècle. Deux massifs forestiers y sont couramment

\footnotetext{
${ }^{8}$ Discipline participant à l'étude des environnements anciens, la palynologie se base sur des analyses consistant en un comptage des pollens et des spores piégés dans les sédiments anciens, qu'il s'agisse de niveaux archéologiques ou d'une sédimentation naturelle. Les proportions de spores et de pollens rapportées à la datation des couches permettent d'approcher la composition du couvert végétal aux époques anciennes et son évolution dans le temps.

${ }_{9}^{9}$ Archives départementales de la Vendée, 3P/228/1, tableau d'assemblage du cadastre, Saint-Hilaire de Talmont.

${ }^{10}$ Carte consultable en ligne, URL : https://www.geoportail.gouv.fr/donnees/carte-de-cassini (page consultée le 11 août 2018).

${ }^{11}$ Service historique de la défense, $\mathrm{J}^{10} \mathrm{C} 1293$, pièce 19.
} 
évoqués : la forêt de Jard et celle d'Orbestier. La première mention de cette dernière se trouve dans la charte de fondation de l'abbaye de SaintJean d'Orbestier par Guillaume, duc d'Aquitaine et comte de Poitou en $1107^{12}$, confirmée et amplifiée par Richard Cour de Lion en $1182^{13}$. Ce document fournit le premier état des lieux exploitable de la situation et de l'étendue de cette forêt. La donation concède à l'abbaye le lieu d'Orbestier et les terres cultivées et incultes, vignes, bois, forêts situées sur la côte. Bien que les noms de lieux soient difficiles à retrouver, le paysage décrit pourrait correspondre à une étendue située approximativement entre la rivière de la Vertonne au nord, la mer à l'ouest et Port Juré au sud-est. En effet, le texte incorpore dans la donation un ensemble de rivières et de petits fleuves côtiers se jetant dans la mer, encore reconnaissables dans 1 'hydrographie actuelle ${ }^{14}$. De cette étendue, la foresta comtale proprement dite n'occupe qu'une partie, puisque la foresta de Orbisterio est distinguée du reste et n'est pas concédée en propre aux moines. D'autre part, des bois, landes maritimes et autres brandes sont également citées parmi les terres incultes qui leur sont concédées. Le terme de foresta ne s'applique pas spécifiquement aux espaces boisés à cette époque, mais à toute une variété de terres incultes ${ }^{15}$. Il semblerait alors que la forêt comtale d'Orbestier désigne dans cet acte un territoire réservé à l'usage du comte. Les documents plus tardifs ne rappellent pas systématiquement ses limites exactes. La forêt est mentionnée comme un ensemble dans la confirmation des privilèges de l'abbaye d'Orbestier par le vicomte Guy de Thouars en $1272^{16}$. Au

\footnotetext{
12 «Cartulaire de l'abbaye d'Orbestier », éd. Louis de la Boutetière, Poitiers, Archives Historiques du Poitou, t. 6, 1877, n¹, p. 1 .

${ }^{13}$ « Cartulaire de l'abbaye d'Orbestier », éd. cit., n 4, p. 6.

${ }^{14} \mathrm{Il}$ s'agit du torrens Illicum et du torrens qui defluit per prata Ogerii, que l'on peut assimiler d'après leur situation au Payré et au Ruisseau du Puits, qui se jettent dans la mer à environ dix kilomètres de distance sur la côte. Voir la carte IGN Série Bleue 1227 OT, « Les Sables d'Olonne » (éch. 1/25 000).

${ }^{15}$ Roland Bechmann, Des arbres et des hommes, op. cit., 1984, p. 25.

16 « Cartulaire de l'abbaye d'Orbestier », éd. cit., n 71, p. 80.
} 
$\mathrm{XIV}^{\mathrm{e}}$ siècle, l'existence d'espaces boisés est confirmée par quelques actes relatifs à la coupe du bois. Il faut attendre le début $d u X V^{\mathrm{e}}$ siècle pour retrouver une mention de l'étendue de la forêt d'Orbestier. En 1411, a lieu une transaction entre Pierre d'Amboise, seigneur de Talmont, et l'abbaye de Saint-Jean d'Orbestier ${ }^{17}$. À cette occasion est dressé un récapitulatif de toutes les donations successives de terres et de rentes à l'abbaye. En 1444, une lettre patente de Charles VII qualifie la forêt d'Orbestier de « belle et grant forest », sans plus de précisions ${ }^{18}$. Ce sont finalement les comptes de la forêt de Talmont de la seconde moitié du $\mathrm{XV}^{\mathrm{e}}$ siècle qui nous fournissent les informations les plus fiables à ce sujet $^{19}$. Les paroisses concernées sont toutes situées à l'ouest et au nordouest de Talmont, ce qui rejoint la cartographie d'époque moderne.

Quant à la forêt de Jard, le peu de documentation disponible rend ses contours difficiles à évaluer. Les archives de l'abbaye prémontrée de Lieu-Dieu en Jard n'ont malheureusement pas fait l'objet d'une édition ni même d'un classement. Quelques mentions issues des archives des abbayes locales permettent toutefois de localiser cette forêt au sud de Talmont, sur le territoire de la commune actuelle d'Angles : en 1207, les chanoines de Lieu-Dieu et les moines du prieuré de Fontaines, à Angles, s'accordent sur la prise de bois de chauffage en forêt de Jard, sur laquelle les chanoines ont la haute main ${ }^{20}$. La part respective de l'arbre et des autres espaces incultes, comme les marais ou les landes, reste indéterminée pour cet espace, tout comme pour la forêt d'Orbestier.

La forêt médiévale de Talmont correspond ainsi à deux ensembles distincts et écologiquement composites, bois, landes et zones humides.

\footnotetext{
${ }^{17}$ « Cartulaire de l'abbaye d'Orbestier », éd. cit., n 285 p. 385

18 « Cartulaire de l'abbaye d'Orbestier », éd. cit., n 325 p. 483.

${ }^{19}$ Archives départementales de la Vendée, 1E/1265.

${ }^{20}$ Archives départementales de la Vendée, H/183 (liasse).
} 
C'est finalement au travers des vestiges de leur exploitation que ces territoires apparaissent le plus clairement. L'énumération exhaustive en serait interminable; limitons-nous à quelques exemples de la diversité des usages de la forêt de Talmont au Moyen Âge.

\section{Les usages de la forêt de Talmont : quelques exemples}

Les traces laissées par l'exploitation de la forêt de Talmont sont multiples et se retrouvent dans l'intégralité des sources à notre disposition. La forêt fait l'objet d'une exploitation intégrée à tous les aspects de la vie économique par les différents habitants du Talmondais. Un bilan quantitatif portant sur près de quatre siècles s'avère impossible à dresser, tant en raison de la typologie des sources que de leur état souvent fragmentaire ; nous ne pouvons, pour l'heure, ne donner qu'un aperçu de l'omniprésence de la forêt dans la vie économique et sociale du Talmondais médiéval.

\section{Le bois}

Le droit d'usage des forêts talmondaises le plus couramment cité est le droit de se fournir en bois, et tout particulièrement en bois de chauffage. Cet usage concerne aussi bien seigneurs et moines que paysans. En effet, la consommation de combustible est considérable dans l'Occident médiéval, aussi bien pour les activités artisanales que pour le chauffage et la cuisson domestique des aliments. La fourniture du bois pour la cuisson du pain est un besoin permanent, tant au château que dans les communautés monastiques ou paysannes, tandis que les moyens de chauffage à faible rendement provoquent une importante consommation de bois. Les règlements, donations et les inévitables conflits entre les divers usagers des espaces forestiers ont cependant engendré une production écrite variée. En 1195, une charte de Richard Cœur de Lion, 
comte de Poitou et seigneur de Talmont, accorde ainsi aux moines du prieuré grandmontain de la Meilleraye, situé au cœur de la forêt d'Orbestier, le droit d'exploiter tout le bois nécessaire selon leur volonté $^{21}$. Le terme de chauffagium, qui désigne le droit coutumier de prélever du bois pour le feu dans les espaces boisés, est omniprésent dans les actes de la pratique. Les dévolutions de droits, temporaires ou permanentes, sur la forêt seigneuriale en font mention. Plusieurs litiges et règlements l'attestent au cours du temps : en 1248, Raoul de Mauléon, seigneur de Talmont, confirme aux moines de Talmont l'usage des bois ad comburendum de branchiis et de arboribus ${ }^{22}$. Le bois des forêts de la seigneurie est ainsi la source exclusive du bois employé sur les chantiers au château de Talmont, du moins pour ce que nous apprennent les sources du XV $\mathrm{XV}^{\mathrm{e}}$ siècle. En 1414-1415, le bois de la forêt d'Orbestier utilisé sur les chantiers de construction de la seigneurie est acheté sous forme de produits semi-finis, planches, lattes et madriers. Le prix payé est faible, une livre et demie, ce qui s'explique par le coût modique du transport entre les bois exploités et le bourg de Talmont ${ }^{23}$. Le bois est enfin employé sous une forme transformée: le charbon. La production de charbon est, au Moyen Âge, extrêmement dispendieuse en bois, nécessitant huit à dix kilogrammes pour un kilogramme de charbon ${ }^{24}$. Cet artisanat est attesté en forêt d'Orbestier à la fin du $\mathrm{XV}^{\mathrm{e}}$ siècle $^{25}$.

\section{Forêt, agriculture et élevage}

Au-delà du prélèvement de matériaux et de ressources en forêt, les espaces incultes eux-mêmes représentent des ressources importantes pour

\footnotetext{
${ }^{21}$ Archives départementales de la Vendée, H/190, n².

22 « Pour brûler branches et arbres » (nous traduisons), « Cartulaire de l'abbaye de Talmont », éd. Louis de la Boutetière, Mémoires de la Société des Antiquaires de l'Ouest, $1^{\text {ère }}$ s., tome 36, 1872, p 41-497, $n^{\circ} 535$ p. 461.

${ }^{23}$ Archives départementales de la Vendée, 1E/1245, fol. 25v-29v.

${ }^{24}$ Roland Bechmann, Des arbres et des hommes, op. cit., p. 187.

${ }^{25}$ Archives départementales de la Vendée, 1E/1265 (1492).
} 
l'agriculture locale. La forêt est largement mise à contribution dans les agricultures des sociétés préindustrielles européennes, parmi lesquelles les sociétés médiévales ne font pas exception. Les terres incultes représentent en effet une réserve de terres arables qui peuvent être défrichées et mises en culture si le besoin s'en fait sentir. Les défrichements, utilisant la force physique ou le $\mathrm{feu}^{26}$, connaissent des rythmes séculaires selon la conjoncture économique et démographique. En Talmondais, la documentation apporte de temps à autre des éclaircissements sur la mise en culture d'espaces jusque-là boisés. En 1444, Louis d'Amboise, seigneur de Talmont, ordonne ainsi à ses officiers de faire dresser l'état des défrichements en cours dans la forêt d'Orbestier afin que l'abbaye d'Orbestier perçoive le cinquième des redevances dues sur ces terres. Ce mandement est renouvelé en $1452^{27}$ et un état des défrichements entrepris depuis huit ans est dressé à cette occasion. On compte alors quatorze pièces de terres différentes cultivées par des tenanciers; la superficie de ces terres s'échelonne entre une et plus de quatre sexterées ${ }^{28}$ de terre. Ces défrichements en forêt sont une illustration de la reconstruction économique de la seigneurie. Fabien Chanson, dans son étude économique et sociale des campagnes du Talmondais au $\mathrm{XV}^{\mathrm{e}}$ siècle, montre que les surfaces utiles augmentent peu dans la première moitié du siècle, avant que la reconstruction démographique et économique ne pousse à coloniser de nouvelles terres,

\footnotetext{
${ }^{26}$ L'agriculture sur brûlis est marginalement pratiquée dans l'Occident médiéval et moderne, plus particulièrement dans les régions côtières de l'océan Atlantique (France de l'Ouest, Îles Britanniques) ; pour un aperçu des techniques de défrichage par le feu à l'époque médiévale, voir Georges Comet, $\mathrm{Le}$ paysan et son outil : essai d'histoire technique des céréales. France: VIII $-X V^{e}$ siècle, Rome, École française de Rome, 1992 ; Jean-Marc Moriceau, Terres mouvantes. Les campagnes françaises du féodalisme à la mondialisation, XII $-X V^{e}$ siècle, Paris, Fayard, 2002.

27 « Cartulaire de l'abbaye d'Orbestier », éd. cit., n 342, p. 527.

${ }^{28}$ Terme dialectal poitevin, superficie de terre qui produit un setier de grains.
} 
incultes ou laissées à l'abandon, à partir des années $1450^{29}$. La forêt de Talmont constitue ainsi une réserve de terres arables pour les paysans. Les différents usagers de la forêt bénéficient également d'un droit d'usage en forêt pour la pâture des bêtes. Les forêts médiévales sont en effet utilisées par les éleveurs préférentiellement aux prairies pour faire paître les animaux domestiques, bovins, ovins, équins et porcins. Le porc a toutefois la première place parmi les animaux élevés en forêt dans de nombreux $\operatorname{cas}^{30}$. En 1229, Raoul, abbé de Talmont, conclut un accord avec les chanoines de l'abbaye de Lieu-Dieu en Jard sur la pâture des porcs en forêt de Jard, en précisant les droits d'usage de chacun des établissements réguliers ${ }^{31}$. D’autres animaux sont également signalés par la documentation à notre disposition. À la fin $\mathrm{du} \mathrm{XV}^{\mathrm{e}}$ siècle, la plus grande partie des comptes de la forêt de Talmont consiste en une liste précise des redevances dues pour la pâture des animaux des paysans locaux en forêt d'Orbestier $^{32}$. En 1468, les redevances pour les porcs sont notées en premier, à raison de deux sous et six deniers pour un an de pâture ; la pâture d'un bœuf ou d'une vache vaut, elle, cinq rais et demi d'avoine. Les modalités matérielles de la pâture des animaux en forêt d'Orbestier sont peu connues. Pourtant, comme le montrent les prospections de terrain réalisées par François Duceppe-Lamarre dans différents massifs forestiers du nord de la France, les structures matérielles médiévales liées à l'élevage forestier ont contribué à modifier les paysages en fonction des conditions naturelles, des besoins des éleveurs et des parcours des

\footnotetext{
${ }^{29}$ Fabien Chanson, Les comptes de la seigneurie de Talmont (1412-1499). Étude économique et sociale du Talmondais à la fin du Moyen Âge, mémoire de Master 2 inédit, Université de la Rochelle, 2007, p. 61.

${ }^{30}$ Roland Bechmann, Des arbres et des hommes, op. cit., p. 146 ; François Duceppe-Lamarre, Chasse et pâturage dans les forêts du Nord de la France: pour une archéologie du paysage sylvestre, $X I^{e}-X V I^{e}$ siècle, Paris, L'Harmattan, 2009.

31 «Cartulaire de l'abbaye de Talmont», éd. cit., n 433, p. 374.

${ }^{32}$ Archives départementales de la Vendée, 1E/1265.
} 
$\operatorname{animaux}^{33}$. Dans le cas des massifs forestiers du Talmondais, seule une étude de terrain comparable pourrait permettre de déceler pareilles structures archéologiques.

\section{Les chasses seigneuriales en forêt de Talmont}

L'importance de la chasse pour l'aristocratie laïque médiévale n'est plus à prouver. La chasse des grandes bêtes sauvages, associée à un mode de vie aristocratique au moins depuis l'Antiquité romaine, est ainsi une facette caractéristique $\mathrm{du}$ comportement seigneurial. François Duceppe-Lamarre qualifie ainsi la chasse médiévale d' "activité de prédation liée seulement à un groupe social», celui de l'aristocratie laïque, maîtresse des châteaux ${ }^{34}$. L'examen des éléments de l'alimentation issus de la fouille du bâtiment nord du château de Talmont a montré que celui-ci ne dérogeait pas et présentait même des proportions exceptionnelles de faune sauvage dans les phases tardives d'occupation, datées du $\mathrm{XV}^{\mathrm{e}}$ siècle ${ }^{35}$. C'est en effet au château que nous retrouvons les vestiges osseux des animaux chassés. Les espèces terrestres sauvages rencontrées dans les niveaux datés du XIII ${ }^{\mathrm{e}}$ au Xv ${ }^{\mathrm{e}}$ siècle sont relativement restreintes : sangliers, cerfs, chevreuils, c'est-à-dire la trilogie du gibier forestier; mais aussi lièvres, lapins, oies sauvages, canards, faisans,

\footnotetext{
${ }^{33}$ François Duceppe-Lamarre, Chasse et pâturage dans les forêts du Nord de la France, op. cit., p. 55.

${ }^{34}$ Voir notamment: La chasse au Moyen Âge. Actes du colloque de Nice (22-24 juin 1979), Nice, Publications de la Faculté des Lettres de Nice, 1980; La Chasse au Moyen Age, société, traités, symboles, dir. Agostino Paravicini Bagliani et Baudouin Van den Abeele, Turnhout, Brepols, coll. «Micrologus' Library», 2000 ; Baudouin Van den Abeele, «Medieval Hunting », dans A Cultural History of Animals in the Medieval Age, dir. Brigitte Resl, 2007, p. 59-79; François Duceppe-Lamarre, «Le seigneur et l'exercice du droit de chasse. Permanences et évolutions d'un pouvoir social et territorial (XII $-\mathrm{XV}^{\mathrm{e}}$ siècle) ", dans Lieu de pouvoir, lieu de gestion. Le château aux XIII $-X V I^{e}$ siècles : maîtres, terres et sujets, dir. Jean-Marie Cauchies et Jacqueline Guisset, Turnhout, Brepols, 2011, p. 167-180, cit. p. 167.

${ }^{35}$ Aurélia Borvon, «Étude des vestiges fauniques du secteur 1 », dans Le château de Talmont (Talmont Saint-Hilaire, Vendée), rapport final d'opération, SRA Pays de la Loire, 2017, p. 69-108.
} 
perdrix, bécasses des bois, courlis cendrés et petits passereaux ${ }^{36}$. De fait, les chasses seigneuriales du Moyen Âge n'ont globalement concerné qu'un nombre restreint d'espèces animales, et notamment les plus gros animaux, prisés par les seigneurs ${ }^{37}$. Parmi les ossements d'animaux sauvages étudiés au château de Talmont, les mammifères dominent largement, tant en termes de nombre que de poids des restes. Cet état de fait est comparable à celui des contextes seigneuriaux du second Moyen Âge étudiés dans le nord de la France, dans lesquels la primauté des mammifères est visible autant dans les assemblages fauniques que dans la documentation textuelle ${ }^{38}$; la faune aviaire y occupe toutefois une place non négligeable, comme cela est également le cas à Talmont. L'étude archéozoologique montre, elle, une certaine évolution dans la répartition des grandes espèces forestières chassées à Talmont entre le $\mathrm{XIII}^{\mathrm{e}}$ et le $\mathrm{XV}^{\mathrm{e}}$ siècle. Le sanglier domine au XIII ${ }^{\mathrm{e}}$ siècle ; entre la fin du $\mathrm{XIII}^{\mathrm{e}}$ et la fin du XIV $\mathrm{X}^{\mathrm{e}}$ siècle, le nombre de restes de sanglier et de cerf est équivalent. Enfin, entre la fin du XIV et le $\mathrm{XV}^{\mathrm{e}}$ siècle, le nombre de restes de cerf est désormais très important et dépasse largement le nombre de restes de sanglier. Le cas de Talmont n'est pas isolé. Sur la plupart des sites élitaires laïcs de la fin du Moyen Âge, les cervidés sont le gibier le plus abondamment découvert ${ }^{39}$.

Cette répartition est peut-être représentative d'une évolution culturelle touchant les pratiques de chasse à partir du XIII ${ }^{\mathrm{e}}$ siècle dans tout

\footnotetext{
${ }^{36}$ Aurélia Borvon, « Étude archéozoologique du château de Talmont Saint-Hilaire (Vendée), première approche ", dans Le bâtiment nord du château de Talmont Saint-Hilaire, rapport de fouille triennale 2013-2015, SRA Pays de la Loire, 2016, p. 95-106.

${ }^{37} \mathrm{Au}$ sujet de la symbolique de la mise à mort des grands animaux, voir Alain Guerreau, «Les structures de base de la chasse médiévale », dans La Chasse au Moyen Âge, Société, traités, symboles, op. cit., p. 25-32 ; Marylène Patou-Mathis, Mangeurs de viande, de la préhistoire à nos jours, Paris, Perrin, 2017.

38 Robert Delort, «Quels animaux les seigneurs chassaient-ils dans les forêts à la fin du Moyen Âge? ", dans Le château, la chasse et la forêt, Bordeaux, Sud-Ouest éditions, 1988, p. 165-171, cit. p. 167.

39 Benoît Clavel et Sébastien Lepetz, « De la chasse au lièvre à la chasse au cerf, évolution des pratiques de l'âge du fer au Moyen Âge », Archéopages, n² 28, 2009, p. 38-43, cit. p. 39.
} 
l'Occident médiéval, et tout particulièrement en France : le cerf, gibier royal et princier, animal christologique valorisé par l'Église est désormais le gibier noble par excellence tandis que le sanglier, favori des tables romaines et de celles du haut Moyen Âge ${ }^{40}$, est désormais considéré comme un animal diabolique ; sa chasse ne disparaît certes pas, mais elle n'est plus la favorite des grands ${ }^{41}$. Ce propos général doit bien entendu être nuancé vis-à-vis des données issues du terrain. Dans l'état 9 du château de Talmont, daté du $\mathrm{XV}^{\mathrm{e}}$ siècle, le nombre de restes de sanglier retrouvé reste très important. Les données ostéologiques montrent ainsi que les pratiques de chasse des seigneurs de Talmont s'inscrivent fondamentalement dans les normes culturelles observables dans l'Occident médiéval contemporain. La chasse du seigneur de Talmont à la fin du XIII ${ }^{\mathrm{e}}$ siècle, aussi bien celle du porc sauvage que du cerf, est celle des grands animaux forestiers, qui nécessite de vastes étendues parfois aménagées.

Cet aperçu des tableaux de chasse des seigneurs de Talmont à la fin du Moyen Âge doit en effet nous conduire hors de l'enceinte castrale, dans les forêts et les espaces incultes où vivent ces animaux. Les espèces consommées font l'objet de pratiques de chasse différenciées, d'après ce que nous apprennent les actes de la pratique, les textes littéraires et les traités de chasse. Les chasses du second Moyen Âge sont ainsi menées selon deux procédés complètement différents : vénerie et fauconnerie, c'est-à-dire chasse au chien et chasse à l'oiseau ${ }^{42}$. Les grands mammifères forestiers sont chassés «à force» ou «à courre ». Il s'agit d'une chasse mobile, en milieu boisé pour laquelle on utilise les chiens.

\footnotetext{
40 Frédérique Audouin-Rouzeau, «L'alimentation carnée dans l'Occident antique, médiéval et moderne. Identités culturelles et régionales à travers le temps », dans Histoire et identités alimentaires en Europe, Paris, Hachette, 2002, p. 77-100, cit. p. 83.

${ }^{41}$ Michel Pastoureau, "La chasse au sanglier : histoire d'une dévalorisation (IV $-X I V$ siècle) », dans La Chasse au Moyen Âge. Société, traités, symboles, op. cit., p. 7.

${ }^{42}$ Alain Guerreau, « Les structures de base de la chasse médiévale », art. cit., p. 26.
} 
Les textes portent parfois le témoignage de ces chasses seigneuriales dans les forêts du Talmondais. Une charte de décembre 1279 règle un conflit entre les chanoines de Lieu-Dieu en Jard et le vicomte Guy de Thouars, seigneur de Talmont, au sujet du droit de chasse de ce dernier dans les parties des forêts de Jard et d'Orbestier possédées par l'abbaye ${ }^{43}$. Pendant ses chasses, lorsqu'il poursuit un animal, le seigneur de Talmont revendique le droit de «suirre et prendre la dite beste en la dite forest et en la terre à l'abbé et au convent de Jart et en faire nostre voulenté », ce que contestent l'abbé et le convent de Jard. Ce texte recèle de nombreuses précisions sur les réalités concrètes de la chasse aux animaux forestiers en cette fin du XIII ${ }^{\mathrm{e}}$ siècle, telle que pratiquée par les seigneurs de Talmont. La traque n'est pas seulement l'affaire du seigneur mais aussi celle de ses suivants, invités et de son équipage de chasse : il est fait mention du veneur, "qui la dite beste pour nous ou pour nostre commandement chaceroit et suiroit ». Ces veneurs et chasseurs seigneuriaux ont pour tâche principale de mener les chiens de chasse; le règlement de la contestation implique que le veneur doive « retraire ses chiens » avant qu'ils n'entrent sur le territoire de l'abbaye. En effet, si les chiens saisissent et tuent la bête dans la forêt de l'abbaye sans que le veneur ne puisse les en empêcher, les chanoines garderont la bête. Ce texte met aussi en évidence l'importance des chiens dans la chasse des animaux sauvages. Ils sont en effet les principales armes employées pour abattre les animaux, combinés à l'arc ou à l'épieu de chasse. Le texte ne précise pas quels animaux sont chassés de la sorte, mais nous pouvons avancer qu'il s'agit du sanglier ou des cervidés.

La vénerie se caractérise non seulement par l'emploi des chiens de chasse mais également par celle d'un accessoire sonore : la corne de

\footnotetext{
${ }^{43}$ Recueil des documents concernant le Poitou contenus dans les registres de la Chancellerie de
} France, éd. Paul Guérin, Poitiers, Imprimerie Houdin, 1881, t. 1, 172, p. 417. 
chasse, ou corne d'appel, également appelée " olifant» lorsqu'elle est fabriquée en ivoire ${ }^{44}$. Il s'agit d'un outil signifiant, qui « fait apparaître le chasseur comme tel» pour reprendre ici la formulation d'Alain Guerreau ${ }^{45}$. La corne est un moyen employé pour communiquer à distance selon des codes sophistiqués, mais également pour imposer la présence de la chasse seigneuriale dans l'espace sonore de la forêt et de la campagne. Le seigneur, via les cornes de chasse, marque les paysages et les esprits, y compris ceux qui ne peuvent le voir, à une distance bien plus vaste que celle parcourue effectivement par l'équipage seigneurial. Deux exemplaires de telles cornes d'appel ont été découverts dans les niveaux archéologiques du bâtiment nord du château de Talmont, dans des contextes datés du XIII ${ }^{\mathrm{e}}$ et du XIV ${ }^{\mathrm{e}}$ siècle $^{46}$. Ils attestent de leur usage dans les pratiques de vénerie. Ces mobiliers sont les éléments de culture matérielle les plus étroitement liés à la pratique de la chasse qui ont jusqu'alors été découverts au château de Talmont. De fait, les armes de trait découvertes au château ne peuvent pas avec certitude être reliées au monde de la chasse ou à celui de la guerre.

L'autre forme de chasse pratiquée dans l'Occident médiéval est également attestée au château de Talmont: il s'agit de la chasse à l'oiseau, ou fauconnerie. Contrairement à la chasse aux chiens, elle est une chasse statique, pratiquée en milieu découvert. Hommes et femmes peuvent y participer, alors que la vénerie est une activité masculine par essence. Elle concerne avant tout le petit gibier, oiseaux d'eau et petits

\footnotetext{
${ }^{44}$ Sur les cornes de chasse en ivoire, voir Paolo De Vingo, « Le travail de l'os, de la corne et de l'ivoire animaux au cours des premiers siècles du Haut Moyen Âge. Quelques exemples de nouvelles productions du royaume franc », Archéologie médiévale, vol. 39, 2009, p. 17-30. Sur quelques cornes de chasse en céramique découvertes dans l'ouest de la France, Sarah Grenouilleau et Yves Henigfeld, « De la cuisine à la table : aspects de la vaisselle en céramique dans les Pays de la Loire du XI au XVI ${ }^{\mathrm{e}}$ siècle, un premier état de la question », dans Les cuisines, Rennes, CTHS, 2016, p. 115-128.

45 Alain Guerreau, "Chasse», dans Dictionnaire raisonné de l'Occident médiéval, dir. Jacques Le Goff et Jean-Claude Schmitt, Paris, Fayard, 1999, p. 170.

${ }^{46}$ Sarah Grenouilleau, "Étude du mobilier céramique », dans Le château de Talmont Saint-Hilaire (Vendée), rapport final d'opération, SRA Pays de la Loire, 2014, p. 45-65, cit. p. 50.
} 
mammifères comme le lièvre. La présence des oiseaux de proie au château est attestée par la découverte d'ossements de faucon, d'épervier et d'autour des palombes ${ }^{47}$. Il semble que les seigneurs de Talmont aient été fournis en oiseaux de proie capturés dans le milieu naturel par leurs vassaux, au titre des obligations féodales. En témoigne le registre féodal de la seigneurie, à la fin du XIII ${ }^{\mathrm{e}}$ siècle, qui contraint les détenteurs de fiefs en forêt de Jard à apporter au seigneur de Talmont tout faucon ou épervier capturé ${ }^{48}$.

\section{Les défens : bornage et lieux réservés en forêt de Talmont}

Une bonne partie des écrits qui documentent la forêt et la chasse à Talmont entre le $\mathrm{XIII}^{\mathrm{e}}$ et la fin $\mathrm{du} \mathrm{XV}^{\mathrm{e}}$ siècle provient d'actes de la pratique, qu'il s'agisse d'actes solennels rappelant les droits du seigneur ou d'une abbaye ou de conflits entre différentes parties. Un sujet revient fort souvent: celui des parties de forêt réservées à l'un ou l'autre propriétaire. Les confirmations des droits des abbayes de Talmont et d'Orbestier contiennent notamment des limitations du droit de prendre bois et d'exploiter la forêt dans un certain nombre de lieux. En 1248, la confirmation des privilèges de l'abbaye de Talmont sur la forêt d'Orbestier comprend des usages sur la forêt, qui ne s'appliquent pas dans un certain nombre de lieux mentionnés ${ }^{49}$. Quelques années plus tard, en 1255, la confirmation des privilèges de la même abbaye par Aimery de Thouars contient exactement les mêmes dispositions, à ceci près que les toponymes y sont latinisées ${ }^{50}$. Que désignent exactement ces lieux réservés par le seigneur? Les mots ont ici leur importance. Ces lieux sont désignés collectivement sous le nom de defensi; la sémantique même du

\footnotetext{
${ }^{47}$ Aurélia Borvon, « Étude des vestiges fauniques du secteur 1 », art. cit., p. 92.

${ }^{48}$ Archives nationales, $1 \mathrm{AP} / 2258$, fol. 17r.

49 « Cartulaire de l'abbaye de Talmont», éd. cit., n 535, p. 462.

50 « Cartulaire de l'abbaye de Talmont», éd. cit., n 536, p. 464.
} 
latin montre qu'il s'agit de lieux fermés, défendus. Les défens correspondent en effet à la matérialisation de l'appropriation des espaces forestiers par l'aristocratie, surtout laïque, au second Moyen Âge. Ils peuvent être définis comme des portions plus ou moins importantes d'espaces forestiers transformés en territoires uniquement réservés à l'usage seigneurial ; à la lumière des données précédemment évoquées et des exemples contemporains, nous pensons bien évidemment aux activités de chasse. Cela implique que les autres usagers en sont exclus : dans tous les actes évoquant ce sujet dans le Talmondais médiéval, les usages coutumiers de la forêt y sont interdits. Encore en 1454-1455, le compte de la forêt de Talmont ${ }^{51}$ indique que les hommes et femmes qui paient le bûchage peuvent « prandre en lad. fourest tout boais sec et mort pour chauffaige sauve es deffends », ce qui indique la pérennité de cette forme de division des espaces forestiers. Les défens, et autres formes de cantonnement construits comme les parcs ou les garennes ${ }^{52}$, sont une manifestation matérielle de la domination seigneuriale sur l'espace forestier, dans lequel la circulation des hommes et des bêtes dépend du bon vouloir du maître de la seigneurie. Défens et autres lieux réservés apparaissent ainsi comme les signes privilégiés d'une domestication seigneuriale de la forêt, entendue non seulement comme espace naturel mais aussi comme ressource pour des populations diverses aux objectifs antagonistes. La matérialité du bornage des bois manifeste en dernier lieu la domination seigneuriale en cette matière.

L'étude de la forêt de Talmont sur ces quelques siècles de la fin du Moyen Âge donne à voir un milieu largement construit, exploité et

\footnotetext{
${ }^{51}$ Archives départementales de la Vendée, 1E/1265, fol. 3v.

${ }^{52}$ Elisabeth Zadora-Rio, « Parcs à gibier et garennes à lapins : contribution à une étude archéologique des territoires de chasse dans le paysage médiéval », Hommes et Terres du Nord, vol. 2, n¹, 1986, p. 133-139.
} 
domestiqué par le travail des hommes. L'histoire et l'archéologie trouvent à chaque pas les indices de multiples mises en valeur de la forêt. Les forêts et les espaces incultes sont les espaces où s'expriment plus particulièrement le mode de vie aristocratique et l'emprise des maîtres du château de Talmont sur le territoire de la seigneurie. Mettre en relation les pratiques de consommation au château et l'environnement forestier nous conduit à envisager la forêt en dehors de ses limites strictes : le château de Talmont, les défens et autres cantonnements qui aménagent les forêts et en contraignent l'usage forment un véritable continuum qui matérialise la prégnance du cadre seigneurial sur des espaces supposément incultes et sauvages. Il semble pertinent de ce fait, notamment grâce à l'examen de la culture matérielle et des structures archéologiques, aussi bien que de la documentation textuelle, de considérer la matérialité de la domestication de la forêt, complémentaire de son aspect juridique et territorial lié à la seigneurie. Dans cette optique, le site castral doit impérativement être mis en relation avec les multiples aménagements forestiers, desquels il ne constitue in fine que la pièce maîtresse et le point de cristallisation. Il faut cependant remarquer que cet espace inculte, même s'il est largement anthropisé, a une place spécifique et une importance directement liée à son statut semi-domestiqué dans l'économie et la vie rurale du Talmondais médiéval. La domestication médiévale de la forêt de Talmont a ainsi pour caractéristique de conserver les cultures et les espaces incultes côte à côte au sein du même agrosystème. Cela est également une conséquence des pratiques sociales des élites seigneuriales, pour qui la chasse représente un marqueur social et territorial essentiel, ce qui ne semble pas être le cas au cours des siècles suivants, marqués à partir du $\mathrm{XVI}^{\mathrm{e}}$ siècle par l'embocagement du paysage. Les époques plus récentes ne préservent pas complètement ces pratiques culturales et ces manières d'aménager le territoire. Contrairement au bocage, la forêt médiévale de 
Talmont apparait bien comme un territoire dont l'homme tire profit sans lui retirer totalement sa part sauvage. À l'échelle de ce petit territoire, cet équilibre fragile semble être spécifique à l'époque médiévale. D'autres études monographiques des régions d'Europe marquées par l'embocagement à la même époque permettraient d'élargir cette problématique du passage d'un système agro-paysager à un autre, où la nature semi-domestiquée a laissé la place à des cultures semi-ensauvagées, où le seul souvenir de la forêt est la haie séparant les parcelles. 
